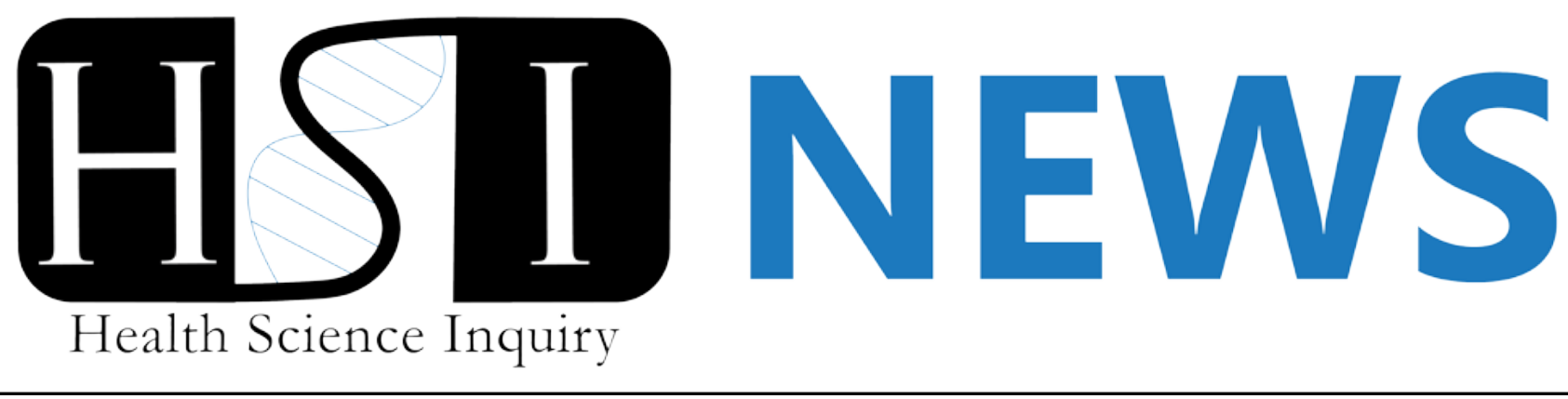

\title{
Persistent organic pollutants (POPs): a sneaky culprit of chronic disease
}

\author{
-Daniel Robinson
}

$\mathbf{T}$ he cause of chronic disease has long been thought to be directly related to genetic predisposition that is, a person's genetic material being the sole factor required to develop chronic disease. As research in the field progressed, a more apparent link between environmental factors and genetics has emerged. Today, we understand chronic disease as an interplay between both genetic predisposition and environmental exposure, where 'genetics loads the gun but environment pulls the trigger' [1].

There are multiple environmental factors that are known to directly contribute to chronic disease. Some better-known examples include lifestyle habits such as diet and exercise in obesity, or the effects of smoking on cardiovascular and lung health. There are also various classes of chemical compounds found within our surroundings that play a critical role in the onset of chronic disease.

One emerging contributor to chronic disease are chemicals known as Persistent Organic Pollutants (POPs). These fatsoluble compounds have been linked to a series of chronic diseases ranging from metabolic issues to carcinogenesis [2]. Regarding the biological basis of disease, it is thought that the structural resemblance between POPs and some hormones produced by our bodies allows POPs to bind and activate hormone receptors within our bodies. This disrupts hormonal balance and endocrine systems which leads to a series of health complications. This is typically manifested through abnormal thyroid functioning, reduced glucose sensitivity, and disruptions in overall energy utilisation, culminating to weight gain and obesity, to diabetes [3], and even to Alzheimer's disease [4].

What is particularly concerning about POPs is their propagated use in many household items: they are present in non-stick compounds that coat kitchen cooking ware (Teflon); they take form as poly-halogenated biphenyl compounds which act as flame retardants used in furniture; and they are the poly-fluorinated compounds used in clothing to repel stains and wrinkles [5]. This widespread use of POPs in many household items in combination with genetic predisposition result in a key interplay that increases the likeliness of developing various chronic diseases.

There are some preventive measures that can be taken to limit exposure to POPs and decrease the likeliness of developing chronic disease. Of primary importance is reducing the number of products that may contain POPs within your household. This means opting for clothing that is not treated with stain-repellents, or furniture that does not contain flame retardants, and having an overall awareness of what constitutes household products. Further, adopting healthy lifestyle and diet habits remains important to reduce the chance of developing chronic disease in general, as reviewed here [6]. Although these measures will not eliminate the risk of developing chronic disease associated with exposure to POPs, it can reduce the general occurrence of chronic disease.

The versatility of POPs has resulted in their presence in many household products, however, given their association with chronic disease means we are using them at a personal and social cost. A continued exposure to persistent organic pollutants will continue to increase the onset of chronic disease. Until stricter regulations regarding the use of POPs in consumer products is introduced, avoiding and limiting exposure to POPs remains a key strategy to prevent chronic disease arising from regular exposure to persistent organic pollutants. 


\section{References}

[1] Suk, W.A., Olden, K., Yang, R.S.H. (2002) Chemical mixtures research: significance and future perspectives. Environ. Health Perspect., 110 (6), 891-892.

[2] Irigaray, P., Newby, J.A., Lacomme, S., Belpomme, D. (2007) Overweight/obesity and cancer genesis: more than a biological link. Biomed and Pharmacother, 61 (10), 665-678.

[3] Lee, D.H., Lee, I.K., Song, K., Steffes, M., Toscano, W., Baker, B.A., Jacobs, D.R. Jr. (2006) A strong dose-response relation between serum concentrations of persistent organic pollutants and diabetes: results from the National Health and Examination Survey 19992002. Diabetes Care, 29 (7), 1638-1644

[4] Hölscher, C. (2011) Diabetes as a risk factor for Alzheimer's disease: insulin signalling impairment in the brain as an alternative model of Alzheimer's disease. Biochem Soc Trans., 39 (4), 891-897.

[5] Sears, M.E., Genuis, S.J. (2012) Environmental Determinants of Chronic Disease and Medical Approaches: Recognition, Avoidance, Supportive Therapy, and Detoxification. J Environ Public Health. doi: 10.1155/2012/356798.

[6] Willet, W.C., Koplan, J.P., Nugent, R., Dusenbury, C., Puska, P., Gaziano, T.A. (2006) Prevention of Chronic Disease by Means of Diet and Lifestyle Changes. In: Disease Control Priorities in Developing Countries. 2nd edition. New York, Oxford University Press.

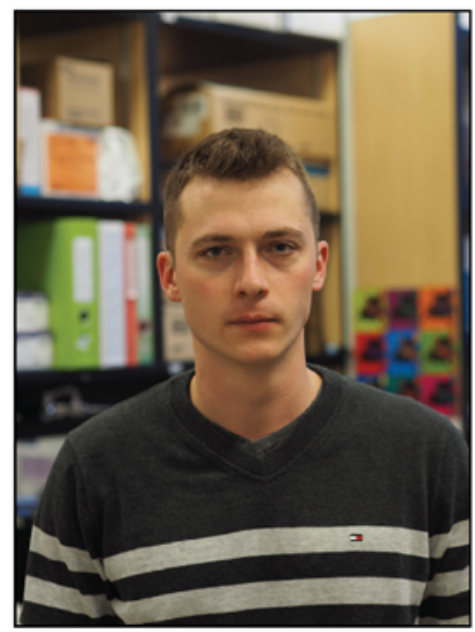

Daniel received his B.Sc. (hons.) in Biochemistry at the University of Manitoba prior to coming to the University of Ottawa to start his doctoral research. He is currently studying gene regulation in adult muscle stem cells during muscle repair to find ways that could be used to stimulate muscle stem cells to repair muscles in persons affected by muscle degenerative disease. When he is out of the lab, Daniel enjoys staying active, going to the gym, and working with the Canadian Army Reserve in his role as a Signals Officer. 